\title{
Research on Countermeasures to the Reform and Development of Physical Education in Ordinary Colleges and Universities from the Perspective of Scientific Fitness
}

\author{
Hao Wang \\ Changzhou vocational institute of textile and garment, Changzhou 213162, China
}

Keywords: scientific fitness, colleges and universities, physical education teaching, reform and development, countermeasures.

\begin{abstract}
With the continuous progress and development of social economy, people's living standards and quality of life have been greatly improved and progressed, and people have become more and more concerned about health. The traditional fitness methods no longer meet people's needs. Scientific fitness has emerged. Scientific fitness is the inevitable combination of the closeness of the people and science and technology, and it is also an inevitable trend of fitness in the future. At present, the education system is constantly reforming and innovating to adapt to social development and progress. Physical education in colleges and universities is the best way to train students in colleges and universities, and it is also the most accessible for college students. Only by doing well in the teaching of physical education in colleges and universities, can lay a good foundation for students in the mentality and habits of fitness, can we better promote the physical and psychological development of college students in the future. However, compared with the requirements of scientific fitness, the author finds that most of the current colleges and universities have certain gaps in terms of teacher's structure and teaching content system, as well as in theoretical teaching and venue equipment. From the perspective of scientific fitness, this article puts forward some countermeasures for the reform and development of physical education in colleges and universities in light of the current status and problems of physical education in ordinary colleges and universities.
\end{abstract}

\section{Introduction}

At present, China is undergoing a reform of the education system. Both the Party Central Committee and the education departments of local governments at all levels attach great importance to this and have formulated relevant regulations and systems for protection. In the document entitled "National Medium and Long-Term Education Reform and Development Program (2010-2020)" issued by the state, the key reference was "taking reform and innovation as a powerful driving force for educational development, reforming teaching content, methods and means, and highlighting the scientific spirit of students, create thinking and innovation." It can be seen that if we want to reform and develop the education system, we must abandon part of the traditional teaching concepts and thinking. We must put the center of the teaching and the focus on the students, so that students can be better all-round development and progress. From the perspective of scientific fitness, this article proposes a scientific solution based on the guiding ideology and knowing theory of the National Medium and Long-Term Education Reform and Development Program, combined with the current situation and problems in current college sports teaching.

\section{Overview of Scientific Fitness}

Scientific fitness, as the name suggests, is scientific fitness, and it is an inevitable combination of the development of science and technology and the development of comprehensive fitness. Fitness exercise can bring people a healthy body, but if the exercise is unreasonable and unscientific, it will bring about potential danger to some extent. Scientific fitness can allow athletes to better master the intensity of physical exercise, exercise time, etc., according to their physical conditions, health, physical strength, age, etc., in combination with the sportsman's living environment and personal 
preferences, habits Such as the development of a reasonable fitness exercise plan, not only can make athletes develop good fitness habits, but also will not bring potential danger to them. Scientific fitness is bound to become the inevitable trend of future fitness. The teaching of college sports must inevitably keep up with the pace of social development. Therefore, teachers should attach importance to scientific fitness and apply it to physical education [1].

\section{Current status and problems of college sports teaching}

(1) Teaching content

At present, the content of physical education teaching in universities in China is mostly based on traditional sports competitions such as football, basketball, volleyball, badminton, swimming, aerobics, martial arts, tennis, etc. The content of teaching is also planned for this sport. Although the project is running, these contents are traditional sports events, and they are not obsolete or eliminated. However, these projects are not loved by all college students and do not meet the personal preferences of some college students. This will lead to some students to college sports teaching. Without interest, it is often perfunctory that it is difficult to achieve the results that sports education should have and it will not help students in their physical and mental development. At the same time, with the advancement and development of the social economy, people's living standards and quality of life have also been greatly improved, and the ideas and thinking of college students who have grown up under this environment have also changed. These traditional sports programs have cannot meet the requirements and vision of college students. In addition, college physical education teachers didn't effectively achieve the basic purpose of physical education when teaching students. In the physical education of colleges and universities, besides imparting students with skills and theoretical knowledge, it is more important to inculcate fitness ideas, answer questions about students' fitness, and cultivate students' interest in fitness. Fitness habit lays the foundation [2].

(2) There are few physical education knowledge lessons, and teaching quality is poor

At present, most physical education courses in colleges and universities, theoretical courses are very rare, less than $10 \%$, some colleges and universities did not even open theoretical courses, only practical lessons. The reasons for this are the following points. The first point is because there are misunderstandings in physical education classes. It is considered that physical education classes only require students to learn practical skills and training, and do not need theory as a guide; the second point is theoretical knowledge of sports. The development of the system is backward, and there is no systematic and perfect theoretical knowledge system. Even if teachers want to impart theoretical knowledge to students, they have no basis and basis. Without theoretical knowledge as a guide, the reform and development of physical education in colleges and universities is seriously hindered. The third point is that teachers pay too much attention to the teaching of practical skills and techniques, and their theoretical knowledge reserves are extremely poor, and they tend to lack theory. The situation has caused serious obstacles in teaching and teaching efficiency and quality are extremely low. It must be understood that theory is the precursor of practice and that thought is the guide for action. Only the theory of perfection, system, and authority can guide the reform and development of college sports teaching.

(3) Fitness equipment and venues are imperfect and old

Most colleges and universities are very imperfect in terms of fitness equipment and venues, and only some of the equipment or venues are also very old. Not only cannot play a good exercise effect, but also cannot guarantee safety, in terms of equipment, many schools only have Traditional tennis, badminton, table tennis and other equipment, and these are not students interested in, and even some schools only have simple horizontal bars, parallel bars, and some even only the playground, physical education teaching can only be carried out outdoors, and carried out Just some simple sports that do not require equipment. In terms of venues, some schools only have simple badminton courts, table tennis venues, football venues and basketball courts, and most of them are outdoors, and this kind of outdoor fitness venue is greatly affected by the season and the weather, which is not conducive to students' fitness. Students are interested in swimming, yoga, aerobics, squash and other emerging 
popular sports are basically unable to teach. Because of the imperfection of the venues and equipment, the physical education cannot meet the students' interests and needs, and the students lose their interest in the physical education classes. How can students cultivate a good habit of scientific fitness? On the other hand, scientific fitness is based on exercise prescriptions as a guide. It is necessary to regularly test students' physical health, cardiopulmonary abilities, and the presence or absence of diseases, so as to adjust the form of exercise, exercise time, and exercise intensity accordingly. Most schools can only detect part of the content, and their detection methods and equipment are very backward and inaccurate. This has caused these schools to be unable to conduct scientific fitness at all. To know that workers must first sharpen their tools for good things, the so-called grinding knife does not mistakenly cut the firewood, therefore, only good equipment and venues as a basis, in order to better carry out scientific fitness exercises, can better promote college sports Teaching reform and development.

\section{The countermeasures for the reform and development of PE teaching in common colleges and universities from the perspective of scientific fitness}

(1) Reform and Innovation of Teaching Content

The oldness and singleness of teaching content will cause students to gradually lose their passion and interest in physical education classes, and gradually produce disgust. Therefore, teachers should make different changes in terms of students' actual conditions, changes in different seasons, influence of weather, and local climate and environment when formulating the teaching content of scientific fitness. Do not confine one or several kinds of sports to compulsory teaching of all students. Instead, according to the students' actual conditions and personal preferences, develop different sports programs suitable for individuals. Sports programs must be more diversified and richer and more rational, so that they can better attract students' interest, so as to better train students to develop good fitness habits. At present, the sports teaching in most colleges and universities are based on traditional sports competitions. However, many students of these traditional sports events are lack of interest. Contemporary college students are more interested in emerging sports programs such as hip-hop, aerobics, yoga, taekwondo, Latin dance, ballroom dancing, etc. However, due to the traditional old-fashioned thinking of colleges and universities, the sports programs that these students are interested in have not been accepted. Therefore, physical education teachers can arrange sports activities that are of interest to these students in elective courses. This will not only meet the growing fitness needs of college students, at the same time, it can also vary from person to person, so that students can choose the sports items that match their interests and interests as much as possible. In addition, when teachers are teaching, it is important to pay attention not to attach too much importance to the transfer of skills and theoretical knowledge, to instill fitness concepts and ideas into students, let students experience the benefits and fun of fitness, and enable students to develop good fitness habits and lay the foundation for the future, even after graduating, fitness exercises can still be performed autonomously, allowing students to reap the benefits of lifetime [3].

(2) Improve the quality of teaching when theoretical classes are added

Scientific fitness should not only actively participate in physical and behavioral activities and follow scientific methods and methods of physical fitness. It must also better understand and value the concepts and concepts of scientific fitness in theory and ideology. Only when the body and mind are put into the science and fitness at the same time can we strengthen the will of scientific fitness, develop good fitness habits, and lay the foundation for the future physical and mental health. Even after graduation, we can still exercise our own fitness. The physical education teaching in colleges and universities should increase the research and improvement of theoretical knowledge and system, and appropriately increase the theoretical class hours so that both the theory class and the practical class can be emphasized. As soon as possible, it will introduce and promote systematic, sound, scientific, and authoritative sports theory knowledge systems so that teachers can rely on and rely on the teaching. On the other hand, in the absence of the theoretical knowledge system, teachers should learn independently, increase their own theoretical knowledge reserves, and use a variety of teaching 
methods and means to teach students, such as using multimedia teaching, micro-video teaching, etc.. Only in this way can college students master theoretical knowledge faster and better, and provide guidance and guidance for their practice and actions.

(3) Increase the investment and maintenance of fitness equipment

Scientific fitness must master the form of exercise, exercise time and exercise intensity, and then use the scientific exercise prescription as a guide for physical exercise and fitness. And these three aspects should be based on medical examination data, according to the athlete's cardiovascular, health, disease, physical strength, age, gender, etc. to determine the specific degree. This requires some modern equipment and equipment to test this aspect. It can regularly detect the basic conditions and changes of college students, and timely adjust the form of exercise, exercise time and exercise intensity. This type of fitness is the most scientific and reasonable [4]. At the same time, in order to meet the personal preferences and requirements of college students, fitness equipment and equipment also need to invest, such as treadmills, rowing machines, body-building vehicles, fitness machines, and so on. A large number and variety of fitness equipment can meet the needs of college students, but also can arouse the interest of students and cultivate students' hobbies and habits. Some female students do not like traditional sports competitions. These equipments can make female students renew their passion for physical education. Not only to cultivate students' good fitness habits, but also to improve the quality and efficiency of physical education teachers. Regular inspection and maintenance of construction equipment should be conducted to prevent accidents.

\section{Conclusion}

Scientific fitness is an inevitable combination and inevitable trend of national fitness and science and technology development. This fitness method is in line with the modern people's pursuit of quality of life, in line with the modern people's fitness concept, in order to better carry out the reform and development of college sports teaching. Therefore, we must pay more attention to scientific fitness. In order to better grasp and promote the use of physical fitness in physical education, it is not only necessary for teachers' abilities, but also for the management personnel and leadership of universities to pay more attention to them and increase their investment in teachers. Educators in colleges and universities should constantly find out the insufficiency of current physical education teaching and improve it according to their own actual conditions, in order to train students to develop good fitness habits, and lay a solid foundation for promoting the reform and development of physical education in colleges and universities.

\section{Acknowledgements}

About the author: Wang Hao (1977.10-) Han, Jiangsu, male, master, lecturer, teacher of Changzhou Textile and Apparel Vocational and Technical College.

Research direction: Physical education and sports training.

\section{References}

[1] Kong Xiangjiu, Song Qiming. Research on the Development of Physical Education Teaching Models in Colleges and Universities in China since the Reform and Opening-up [J]. Sport, 2016(16): 75-77.

[2] Yang Pin. Research on college sports teaching reform and college students' fitness methods [J]. Contemporary Sports Science and Technology, 2016, 6(12): 59-60+62.

[3] YU Xiaoping. Strengthening the Teaching Content of Scientific Fitness in College Physical Education [J]. Contemporary Sports Science and Technology, 2012, 2(11): 46-47.

[4] Fu Lingrong, Li Qiang, Shan Yu. Thoughts on Strengthening Lifelong Fitness Education for All People in Physical Education in Colleges and Universities [J]. Journal of Nanchang College, 1998(01): 18-19. 